\title{
ACTIVATION ENERGIES OF THERMAL ANNEALING OF RADIATION-INDUCED DAMAGE IN n- AND p-CHANNELS OF CMOS INTEGRATED CIRCUITS, Part II
}

\author{
Vitaly Danchenko \\ NASA, Goddard Space Flight Center \\ Greenbelt, Maryland 20771 \\ P. H. Fang \\ Department of Physics \\ Boston College \\ Chestnut Hill, Massachusetts 02167
}

Sidney S. Brashears

Space Science Services

Laurel, Maryland 20810

\section{Abstract}

\section{Experimental}

In Part I of this work, tempering and isothermal curves of annealing of radiation damage in p- and n-channels of RCA CD4007A CMOS integrated circuits were obtained for both the commercial ("soft") and J-process $\left(10^{6}\right.$ rad-hard $)$ fabrication technologies. These experimental data were analyzed for activation energies of thermal annealing using two independent theoretical treatments. Analysis of the activation energy distributions obtained indicated that the radiation-induced charge trapping in the gate oxide occurs mainly around the impurity centers. In this work, the same procedures are applied to devices of RCA's Z-process ( $10^{5}$ rad-hard). Thermal annealing investigation of the Z-process reveals an anomalous annealing behavior of the p-channels, as compared to the p-channels of the commercial and J-processes. The thermal annealing-induced shift of the threshold potential extends far below the original value, which necessitated the development of a new mathematical treatment. This new treatment is presented here.

Introduction

The CMOS (Complementary Metal-Oxide-Semiconductor) technology has proven to be extremely useful in spaceflight electronics due to its compactness, high fan-out capability, low power consumption and great design flexibility. Radiation-induced charge accumulation in the oxide layer caused early CMOS ICs to be extremely radiation-soft $\left(10^{3} \mathrm{Rad}-\mathrm{Si}\right)$, however, in recent years, modification of processing parameters has succeeded in producing devices capable of remaining operative after as much as $10^{6} \mathrm{Rad}-\mathrm{Si}$.

Radiation hardness, however, is not necessarily the prime consideration for CMOS usefulness in space. The processing changes necessary to produce rad-hard devices are sometimes known to affect other device parameters as well, most notably the switching speed (maximum operating frequency) a parameter which is crucial for high-speed onboard data processing and telemetry. Device technologies of intermediate, rather than extreme radiation hardness have accordingly found the widest application for most space applications.

In Part I of the present work, ${ }^{1}$ thermal annealing of radiation damage was investigated in devices of the RCA commercial- (nonrad-hard) and $\mathrm{J}$ - ( $10^{6}$ rad-hard) processes. The annealing data were analyzed using two different theoretical approaches to determine the activation energies of the annealing process. This work (Part II) deals with the RCA Z-process, which is intermediate in radiation hardness between the commercial- and J-process, and has thus found considerably more application in space flight use.

The same method of analysis used in the previous work ${ }^{1}$ is applied here to the Z-process and the results are compared with the results from the other two processes to effect a complete comparison of the thermal annealing characteristics and activation energies of the thermal annealing of radiation damage in the three currently available RCA CMOS processes. A new mathematical treatment was developed, however, for the p-channels of the Z-process, since it reveals an anomalous behavior in the annealing of these devices.

\section{CMOS Integrated Circuits}

The irradiation and annealing described in this work were performed on RCA CD4007A CMOS integrated circuits fabricated using the RCA Z-process $\left(10^{5}\right.$ rad-hard). As with the commercial and Jprocess devices, the only difference between these processes, as reported by the manufacturer, is the nitrogen anneal temperatures:

$\begin{array}{lr}\text { Commercial } & 1100^{\circ} \mathrm{C} \\ \text { Z-Process } & 950^{\circ} \mathrm{C} \\ \text { J-Process } & 850^{\circ} \mathrm{C}\end{array}$

The RCA CD4007A consists of three n-channel and three $\mathrm{p}$ channel enhancement-mode MOS transistors, with one p-and n-pair connected internally as an inverter and separate drain outputs for the other four transistors. The oxide thickness is approximately $90 \mathrm{~nm}$ and is fabricated using the wet-oxide process. Typical gate threshold voltages for the Z-Process are 1.3 to $1.5 \mathrm{~V}$ (n-channels) and -1.6 to $-1.8 \mathrm{~V}$ (p-channels). Gate threshold voltage in this work is defined as that voltage necessary to produce a drain current of $10 \mu \mathrm{A}$ with $10 \mathrm{~V}$ drain-to-substrate voltage. All of the devices came from the same wafer, as made by RCA for the NASA/DoD CREM* program, and are space-flight qualified.

\section{Irradiation}

Irradiation was carried out in the Co-60 Irradiator Room of the Goddard Space Flight Center Radiation Facility. The total dose of Co-60 gamma rays used to produce the radiation damage was $1 \times 10^{4}$ rad-Si, the same dose which was used on the commerical devices in the previous work (the dose for the J-process was $1 \times 10^{5} \mathrm{rad}-\mathrm{Si}$ ). Although, in the previous work, both Co-60 gamma rays and $1 \mathrm{MeV}$ electrons were used, no difference was observed in the annealing between devices exposed to gamma rays and electrons, therefore, in this case, only gamma rays have been used. Two independent dosimetry methods were employed to verify the dose:

(1) A Victoreen model 555 Roentgen Rate Meter with 100X probe, mounted close to the samples in the radiation field.

(2) Nylon radiochromic films, used with a Far West Technology (FWT) Radiochromic Reader, were irradiated at the sample position and read on the reader. The accuracy of calibration of this reader is checked using films which have been irradiated with known doses of gamma rays by the National Bureau of Standards.

*CREM-Component Radiation Effects Measurement experiment is an interagency cooperative program established between NASA, the U.S. Air Force and the Defense Nuclear Agency and is composed of the flight experiment, the ground simulation experiment with monoenergetic radiation sources and the theoretical predictions based on the current models of the space radiation environment. The purpose of the program is to measure radiation effects in semiconductor devices and ICs in the space radiation environment and to compare them with results of the ground simulation experiment and theoretical predictions. Results of one of these experiments, flown on Explorer 55 (Atmosphere Explorer-E) have been published 2,3 
Devices were irradiated in both biased and unbiased conditions. In the biased devices, gate-to-substrate voltages of $+10 \mathrm{~V}$ for the $\mathrm{n}$ channels and $-10 \mathrm{~V}$ for the $\mathrm{p}$-channels were applied. Drain-to-source vol tages applied during irradiation are generally known not to have any effects on the gate threshold.

\section{Annealing}

Annealing of the samples was done in the same Dewar type oven used for the commercial and J-process devices previously reported. As with the commercial and J-process devices, unirradiated control samples were annealed along with the irradiated devices. As expected, the Z-process samples also showed virtually no effect due to the heat treatment alone.

\section{Data Acquisition}

Since it is the most sensitive and reproducible parameter, the gate threshold voltage, $\mathrm{V}_{\mathrm{GT}}$, as defined above, is used as a measure of both radiation damage and annealing. A gate threshold voltage readout and device biasing system built by the University of Michigan for the ground simulation phase of the CREM experiment was used to read out gate threshold voltages before and after irradiation and after each annealing step.

\section{Experimental Results: Tempering and Isothermal Annealing}

After irradiation, the devices were subjected to two different annealing regimes: tempering and isothermal annealing. All annealing was carried out with zero gate and drain bias. In tempering, the temperature is linearly increased as a function of time and the annealing process is interrupted at equal temperature intervals to take readings. In isothermal annealing, the samples are annealed at a constant temperature, the annealing time being carried out at logarithmically increasing intervals.

In order to observe the radiation damage and annealing independent of the small initial variations in $\mathrm{V}_{\mathrm{GT}}$, the data were normalized to unity in the form of an unannealed fraction, defined as:

$$
\mathrm{N}=\frac{\mathrm{V}_{\mathrm{GT}}(\text { after each annealing step })-\mathrm{V}_{\mathrm{GT}} \text { (before irradiation) }}{\mathrm{V}_{\mathrm{GT}}(\text { after irradiation })-\mathrm{V}_{\mathrm{GT}}(\text { before irradiation })}
$$

Tempering

Figure 1 shows the effects of tempering on the p-channels of all three device fabrication technologies; commercial, Z- and J-process. The data for commercial and J-process are from Part I and are shown for comparison with those from the Z-process. The first annealing step is at $50^{\circ} \mathrm{C}$. The temperature was raised at $200^{\circ} \mathrm{C}$ per hour in 25 -degree steps from room temperature (approximately $20^{\circ} \mathrm{C}$ ) to $400^{\circ} \mathrm{C}$ in the case of the Z-process and $450^{\circ} \mathrm{C}$ for the commercial and J-process. The commercial and $\mathrm{J}$-process data represent averages of six samples, three irradiated with Co-60 gamma rays and three with $1 \mathrm{MeV}$ electrons. The Z-process data are averages of two samples irradiated with Co- 60 gamma rays, since it has been observed that there is no difference in the annealing of any of the RCA device technologies as a function of radiation species and the devices, being from the same wafer, are virtually identical in annealing behavior. The root mean square error in $\mathrm{N}$ is approximately $\pm 4 \%$.

The tempering data for the p-channels show virtually no qualitative difference between the biased and unbiased (during irradiation) cases and are therefore averaged together for all three technologies. In the n-channels, however, this is not the case. Figure 2(a) presents the tempering data for unbiased n-channels (commercial, Z- and J-process) and Figure 2(b), the biased n-channels. Although there is almost no difference between biased and unbiased for the commercial devices, a gate bias of $+10 \mathrm{~V}$ during irradiation shows a less significant annealing in both the $\mathrm{Z}$ - and J-process devices, except above $300^{\circ} \mathrm{C}$ where the J-process biased devices begin to anneal more rapidly.

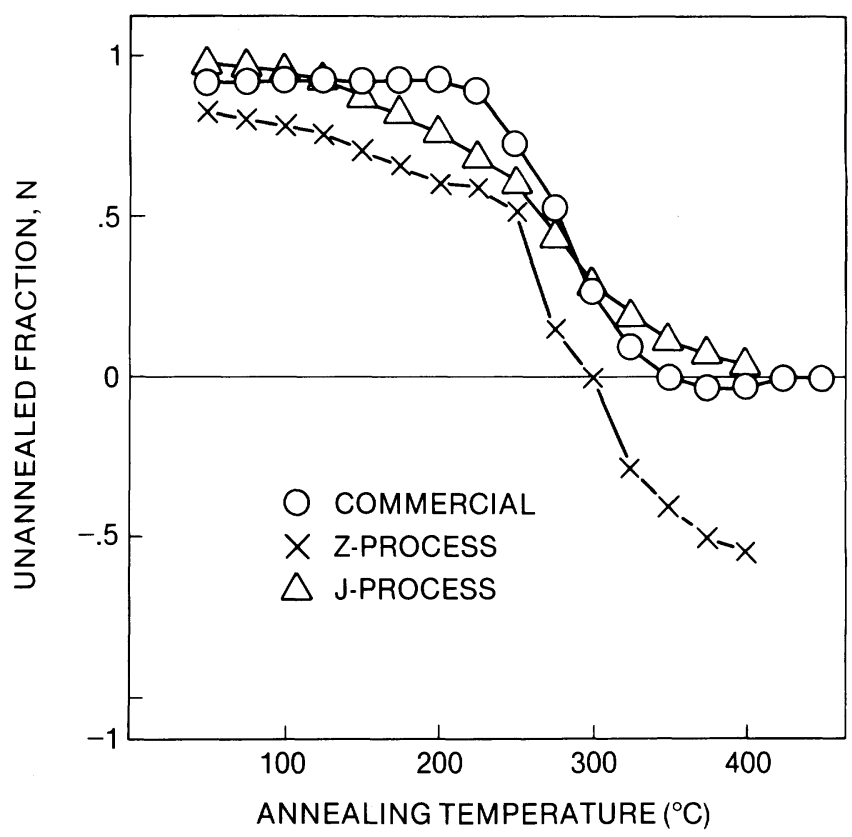

Figure 1. Tempering curves of annealing of radiation-induced damage in p-channels of RCA's commercial (soft), Z- (105 rad-hard) and J(106 rad-hard) process CD4007A CMOS ICs. The data for commercial and $\mathrm{J}$-process are taken from Reference 1.

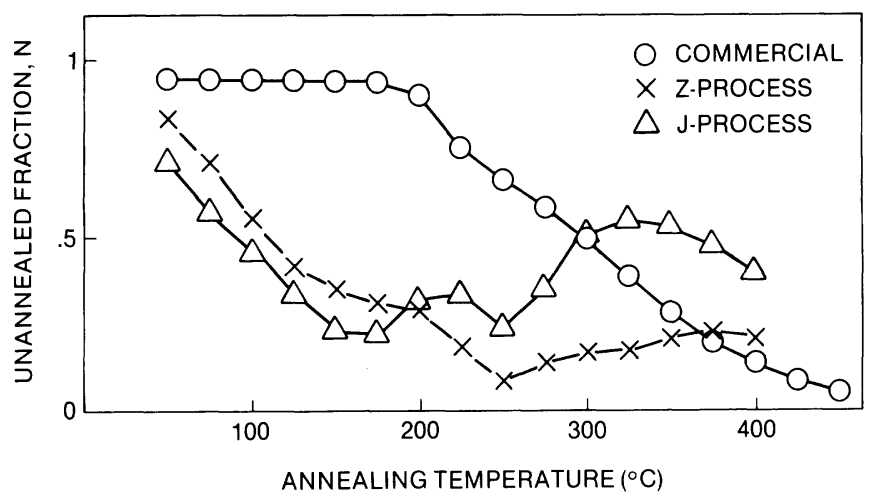

Figure 2(a). Tempering curves of annealing of radiation-induced damage in n-channels unbiased during irradiation of RCA's commercial, Zand J-process CD4007A CMOS ICs. The data for commercial and Jprocess are taken from Reference 1.

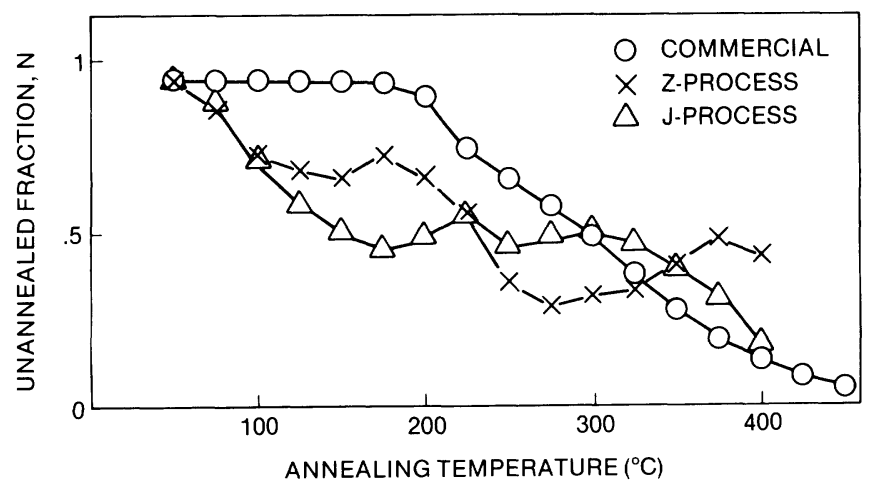

Figure 2(b). Same as Figure 2(a), except n-channels are biased during are biased during irradiation. 
In the p-channel data, the most noticeable difference between the three technologies is that the Z-process devices anneal much below zero, i.e., the gate threshold voltage returns to a lower absolute value than before irradiation. (The usual effect of radiation in CMOS is to shift the n-channel thresholds to a lower absolute value and the pchannel thresholds to a higher absolute value, i.e., more negative in both cases.) The fact that thermal treatment alone does not cause any shift in $\mathrm{V}_{\mathrm{GT}}$ implies that there are two mechanisms at work in this case: one, the removal of trapped positive charge usually associated with thermal annealing of radiation damage in MOS structures, and the other, possibly a radiation-induced electron trapping in the oxide. A similar phenomenon has been observed in p-channel MOS devices manufactured by American Micro-electronics Inc. ${ }^{4}$

\section{Isothermal Annealing}

Isothermal annealing was also performed on the Z-process devices, however, as was pointed out in Part I, activation energy distributions obtained from isothermal annealing data are highly ambiguous due principally to the fact that separate samples are required for each temperature step, thus introducing greater data scattering.

Figure 3 shows isothermal annealing data for the p-channels of the Z-process at three temperatures: $20^{\circ}, 125^{\circ}$ and $250^{\circ} \mathrm{C}$. At $20^{\circ}$ and $125^{\circ} \mathrm{C}$, the difference between biased and unbiased p-channels in their annealing characteristics is negligible, however, at $250^{\circ} \mathrm{C}$, the bias appears to have a very strong effect: devices irradiated under bias anneal below zero an order of magnitude sooner in time than to those irradiated unbiased. This would seem to indicate that the proposed radiation-induced electron trapping is enhanced by a field applied across the gate.

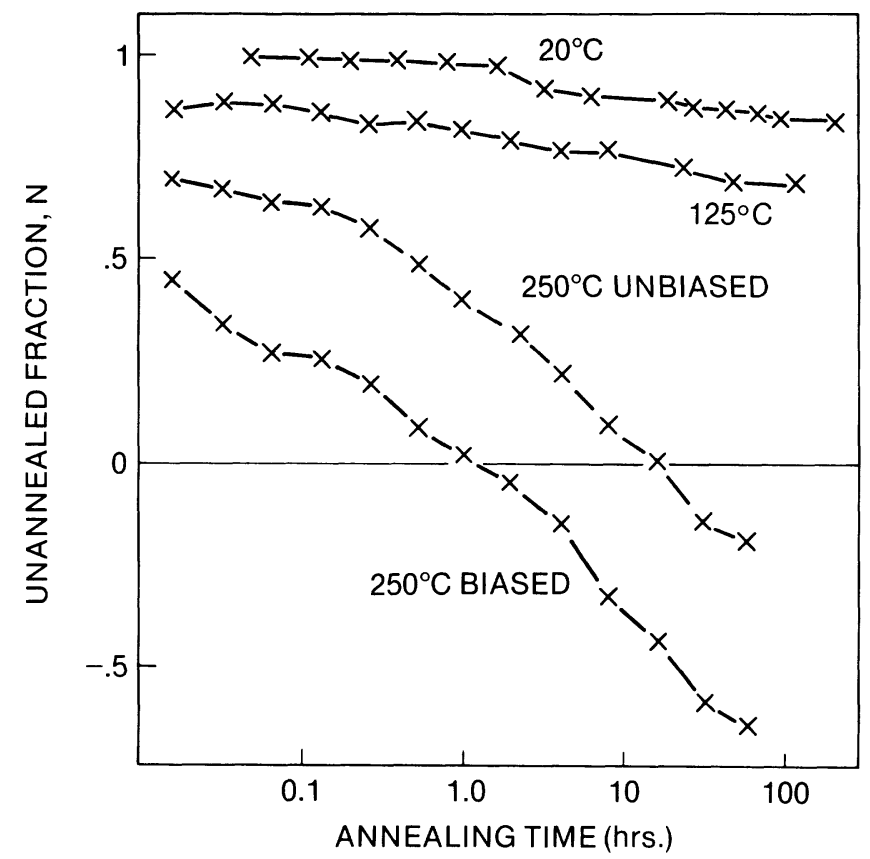

Figure 3. Isothermal curves of annealing of radiation-induced damage in p-channels of RCA's Z-process CD4007A CMOS ICs. The data for $20^{\circ} \mathrm{C}$ and $125^{\circ} \mathrm{C}$ are averages of both biased and unbiased devices.

In the Z-process n-channels, this bias effect is reversed: bias applied during irradiation greatly enhances annealing at room temperature $\left(20^{\circ} \mathrm{C}\right)$ but retards it at elevated temperatures. (Figure 4). In this respect, as well as in general, the behavior of the Z-process n-channels is quite similar to that of the J-process, as reported in Part I.

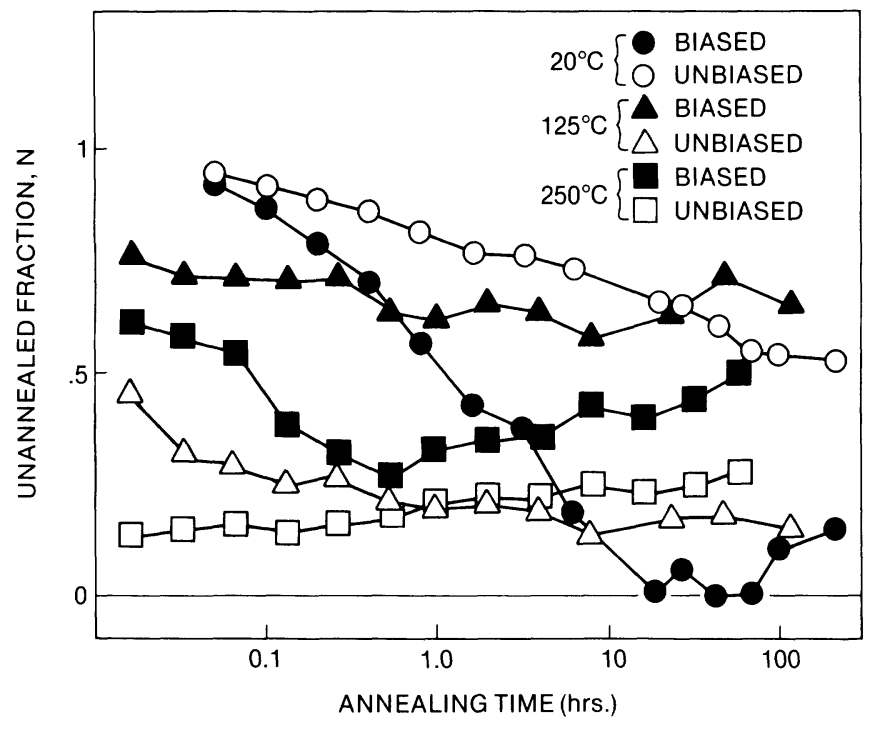

Figure 4. Isothermal curves of annealing of radiation-induced damage in n-channels of RCA's Z-process CD4007A CMOS ICs.

\section{Activation Energy of the Thermal Annealing Process}

Assuming first order kinetics and a distribution of activation energies of thermal annealing, the value of the unannealed fraction remaining after some annealing time, $N(t)$, is given by: 1

$$
N(t)=\int_{0}^{\infty} n_{o}(\epsilon) \exp \left\{-A \int_{0}^{t} \exp \left[-\epsilon / \tau\left(t^{\prime}\right)\right] d t^{\prime}\right\} d \epsilon
$$

where $\tau$, which, in general, may be a function of time, is the product of Boltzmann's constant and the temperature in absolute units, $t$ is the annealing time, $\mathrm{n}_{\mathrm{o}}(\epsilon)$ is the initial (i.e., before annealing) energy distribution of the defect population density, $n_{o}, A$ is an experimentally determined frequency factor and $\epsilon$ is activation energy.

Two methods are used here to determine $n_{o}(\epsilon)$. The first method is a point-by-point method evaluating the derivatives of the $\mathrm{N}$ vs. time or $\mathrm{N}$ vs. temperature data curves. The second method proposes an annealing which can be represented as a sum of two independent processes.

\section{First Method:}

Using the appropriate approximations, ${ }^{1}$ it can be shown that, for tempering, the activation energy is given by the transcendental equation:

$$
\epsilon_{\mathrm{o}} / \tau+\ln \left(\epsilon_{\mathrm{o}} / \tau+2\right)=\ln (\operatorname{Ac} \tau)
$$

where $\mathrm{c}$, the tempering constant, is, for our experimental apparatus, equal to $200^{\circ} \mathrm{C}$ per hour, or, in units applicable to Eq. (3), $2.09 \times 10^{5}$ $\mathrm{sec} / \mathrm{eV}$ and the activation-energy distribution is given by:

$$
\mathrm{n}_{\mathrm{o}}\left(\epsilon_{\mathrm{o}}\right)=-\mathrm{dN} / \mathrm{d} \tau \cdot 1 /\left(\epsilon_{\mathrm{o}} / \tau+1\right)
$$

Equations (3) and (4) were used in the analysis of the tempering data of Figures 1, 2(a) and 2(b) for the Z-process devices to produce the activation energy distributions shown in Figure 5 (p-channels) and Figure 6 (n-channels). In Figures 5 and 6, the activation energy distributions for the Z-process devices are shown superimposed on those of the commercial and $\mathrm{J}$-process for comparison.

The shape of the activation energy distribution curve depends on the slope of the annealing curve rather than its absolute value, hence we see for example, that, despite the extensive annealing below zero demonstrated by p-channels of the Z-process devices, their activation 


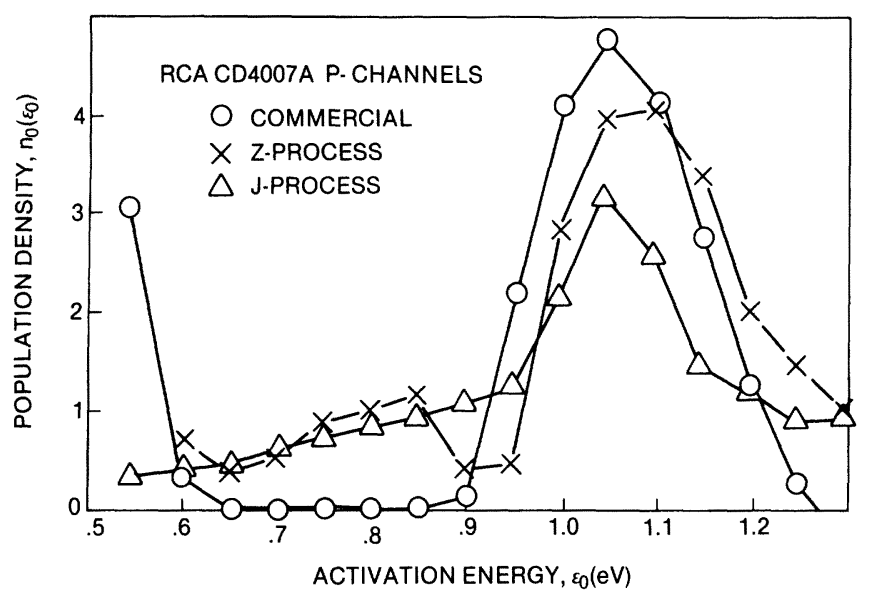

Figure 5. Activation energy distribution calculated from the tempering data shown in Figure 1.

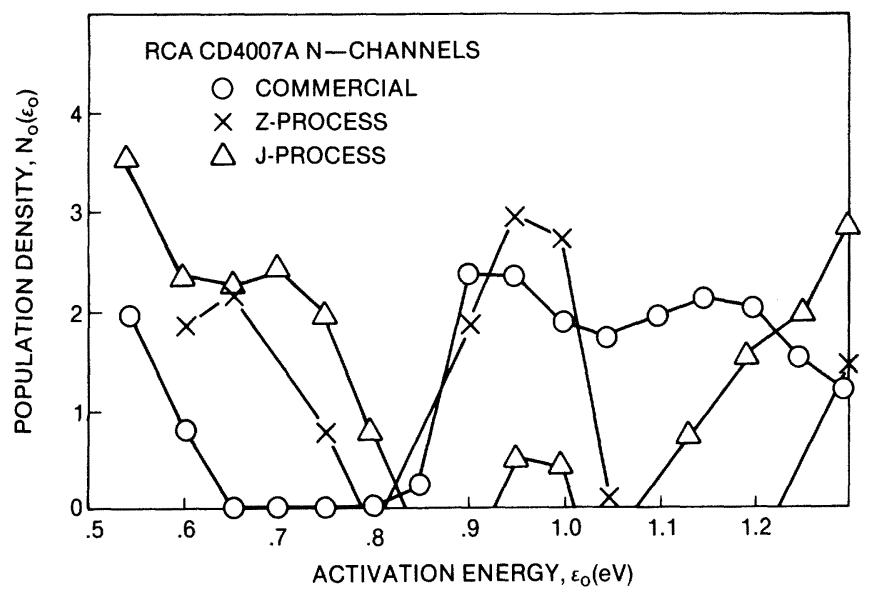

Figure 6. Activation energy distribution calculated from the tempering data shown in Figures 2(a) and 2(b).

energy distribution is actually quite similar to those of the commercial and J-process, as seen in Figure 5.

In the case of the n-channels of the Z-process (Figure 6), the similarity is not as evident at first glance. Although the annealing data are presented for both biased and unbiased devices, both conditions lead to very similar activation energy distributions, since the slopes of both sets of data are similar, as in the case of Fig. 5.

It will be noted, however, that both the $\mathrm{Z}$ - and J-process curves exhibit maxima and minima at approximately the same points on the activation-energy axis, most notably a maximum at approximately 0.95 $\mathrm{eV}$. Further, although the value for the J-process at this point is lower than that of the Z-process, it is higher elsewhere.

It is interesting to consider the area under the curves of Figure 6, i.e.,

$$
\int_{\epsilon_{\mathrm{o}}=0.5 \mathrm{eV}}^{1.3 \mathrm{eV}} \mathrm{n}_{\mathrm{o}}\left(\epsilon_{\mathrm{o}}\right) \mathrm{d} \epsilon_{\mathrm{o}}
$$

which represents the total number of defects (normalized to unity) annealed within this temperature range. These areas are equal to within $<10 \%$, indicating that the total number of defects annealed is the same for each process; only their activation energies of annealing have been shifted. It might be pointed out that if the limits of integration were different, this might not be the case, however, as can be seen from Fig. $7\left(\right.$ a) and $7(\mathrm{~b})$, at $400^{\circ} \mathrm{C}$, the annealing in all cases has gone almost

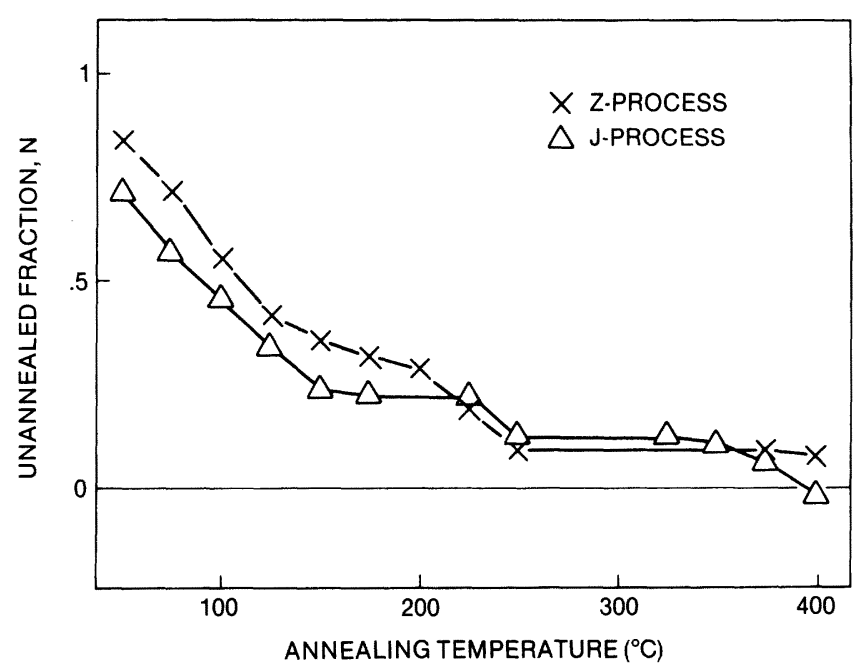

Figure 7(a). Tempering data of Figure 2(a) with reverse annealing removed.

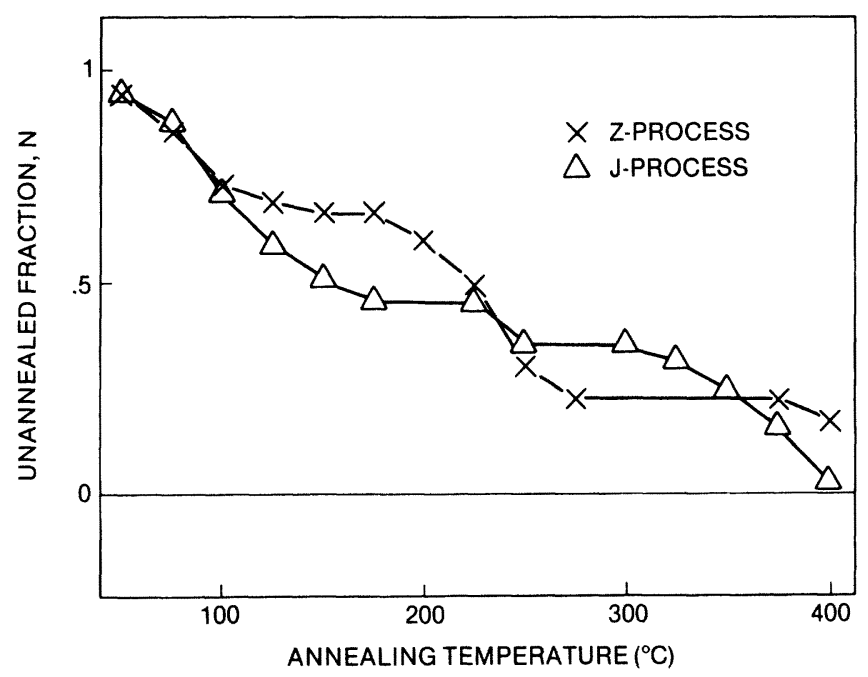

Figure 7(b). Tempering data of Figure 2(b) with reverse annealing removed.

to zero. This would indicate that almost all of the radiation-induced defects have been annealed, and thus the comparison is valid. When this same procedure is applied to the p-channels (Figure 5), the variation is $<15 \%$. It might be argued that, since the damage is normalized to unity initially, the areas under the curves would be expected to be equal, particularly in view of the consideration that, in the case of the n-channels, if only the negative-going parts of the annealing curves are considered, the total annealing is, in the case of the $\mathrm{Z}$ - and J-process, almost equal to the initial damage. In the case of the p-channels, however, the Z-process devices anneal to more than $50 \%$ below zero, which is a much greater variation than is reflected in the activation-energy curves.

The fact that, if the negative-going parts of the n-channel annealing curves are neglected, their annealing proceeds almost to zero (Figure 7) suggests that the negative-going and positive-going parts of the annealing curves in fact represent two independent processes.

In Part I, it was postulated that the double peaks of the activation energy distributions for the n-channels were associated with the double diffusion of phosphorus and boron to form the p-well, in which the radiation-induced charge trapping in the oxide grown from this doublydoped silicon occurs in the vicinity of these impurities. The very large 
annealing below zero observed in the p-channels at $250^{\circ} \mathrm{C}$ would seem to be additional evidence that this is indeed the case, if, as we have proposed, this effect is due to radiation-induced electron trapping.

\section{Second Method:}

\section{(1) Kinetic Equation}

A basic quantity in the kinetic energy is $\mathrm{N}$, related to the density of the defects. In CMOS radiation damage and annealing literature, the definition of $\mathrm{N}$ is given by Eq. 1, where $\mathrm{V}_{\mathrm{GT}}$ (after each annealing step) is the value of the gate threshold voltage after irradiation with a given dose and after annealing for a time t. In CMOS with a heavy doping in the oxide region, a value of $\mathrm{V}_{\mathrm{GT}}$ (after each annealing step) less than $\mathrm{V}_{\mathrm{GT}}$ (before irradiation) has been observed by us and we propose to describe this behavior as an anomalous annealing. ${ }^{4}$ In this case, for a given range of $\mathrm{t}, \mathrm{N}$ would become negative and therefore meaningless. We have thus proposed to apply directly the kinetic equation to the variable $\mathrm{V}_{\mathrm{GT}}$ (after each annealing step), hence, for simplicity, we will call it $\mathrm{V}(\mathrm{t})$. In particular, the following equation has been suggested:

$$
V(t)=\frac{a}{1+\lambda_{1} t}+V_{o}-M_{\infty}\left(1-\frac{1}{1+\lambda_{2} t}\right),
$$

where the symbol a represents a normal annealing process while $\mathrm{M}$ represents a radiation damage assisted annealing and $\lambda_{1}, \lambda_{2}$, their corresponding annealing rate constants, Eq. 6 can be written simply as

$$
\mathrm{V}(\mathrm{t})=\mathrm{V}_{1}+\frac{\mathrm{a}}{1+\lambda_{1} \mathrm{t}}+\frac{\mathrm{b}}{1+\lambda_{2} \mathrm{t}},
$$

with $\mathrm{V}_{1}=\mathrm{V}_{\mathrm{o}}-\mathrm{M}_{\infty}$ and $\mathrm{b}=\mathrm{M}_{\infty}$.

In Ref. $4, V_{1}$, a and $b$ are positive, therefore, $V(t)$ is a monotonic decay function without a maximum or minimum. In the annealing of CMOS made with the Z-process, however, when radiation damage in the n-channel is introduced under a biased condition, the subsequent isothermal annealing, as shown in Figure 8, shows an initial recovery followed by a subsequent reverse annealing, thus a maximum in $\mathrm{V}(\mathrm{t})$ is observed.

Phenomenologically, Eq. (7) can be used to derive the result of Figure 8 when one allows one of the constants, either $a$ or $b$, to be negative. Physically, the radiation introduces positive charges in the oxide and the interface between the oxide and the semiconductor which leads to an increase of the absolute value of gate threshold voltage in the p-channel and a decrease in the n-channel. Therefore, the sign of a and $b$ can be related to the build-up or diminishing of the residual charge traps and/or its associated charge states. For example, if we take $\mathrm{a}$ as positive and $\mathrm{b}$ as negative, then the term containing $\mathrm{a}$, with the rate $\lambda_{1}$, will represent a decreasing $V$ while the term containing $b$, with rate $\lambda_{2}$, represents an increasing $V$. Since the increasing V precedes the decreasing $\mathrm{V}$, one can immediately conclude that $\lambda_{1}$ is smaller than $\lambda_{2}$

A verification of the above physical model could be made by a bias-temperature (B-T) treatment.

\section{(2) Numerical Analysis}

Proceeding with the analysis, we differentiate $V(t)$ of Eq. 5 with respect to $t$ and after a rearrangement, we arrive at an equation for $t^{*}$ a critical $t$ which gives $V(t)$ a maximum, that is,

$$
\mathrm{t}^{*}=(\kappa-1) /\left(\lambda_{1}-\kappa \lambda_{2}\right),
$$

where $\kappa=\left(-a \lambda_{1} / b \lambda_{2}\right)^{1 / 2}$. A necessary condition for the existence of a real and positive $t^{*}$, thus a maximum in $\mathrm{V}(\mathrm{t})$ as in the biased $\mathrm{n}$-channel, is that $\mathrm{a}$ and $\mathrm{b}$ have opposite signs, that is, if $\mathrm{a}$ is positive, $\mathrm{b}$ should be negative. In that case, one can also establish that $\lambda_{1}<\lambda_{2}$. Note that in the n-channel case, an increasing $\mathrm{V}$ represents normal annęaling and

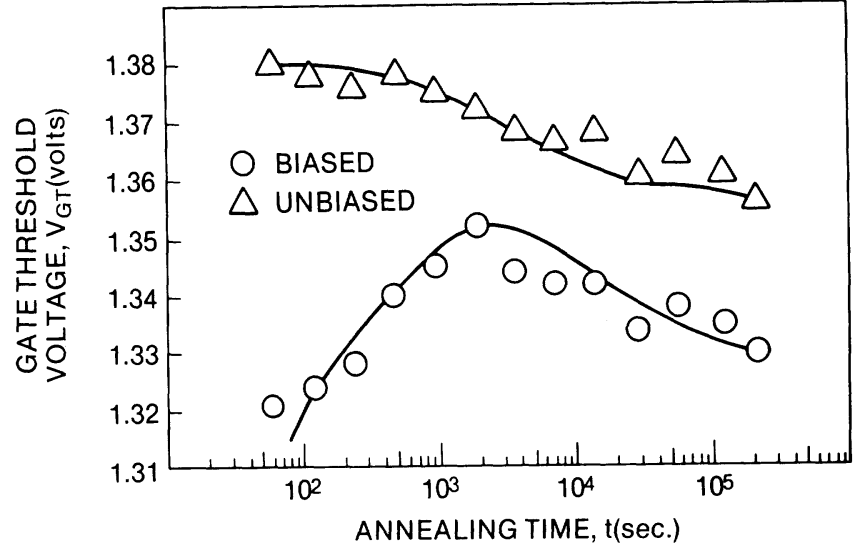

Figure 8. Isothermal $\left(250^{\circ} \mathrm{C}\right)$ annealing data for Z-process n-channels. The solid curve is numerically fitted from Equation (6) and Table 2.

decreasing $\mathrm{V}$ a reverse annealing, while in the p-channel case, the opposite is true. We have the following table for the biased (subscript B) and unbiased (subscript $U$ ) annealing cases:

\section{Table 1. Characteristics of Annealing Parameters}

$\begin{array}{lcccc} & \mathrm{n}_{\mathrm{B}} & \mathrm{n}_{\mathrm{U}} & \mathrm{p}_{\mathrm{B}} & \mathrm{p}_{\mathrm{U}} \\ \mathrm{a} & >0 & >0 & >0 & >0 \\ \mathrm{~b}\left(=\mathrm{M}_{\infty}\right) & <0 & \leqslant 0 & >0 & >0\end{array}$

In all cases, $\lambda_{1}<\lambda_{2}$ for the sake of a consistent analysis.

To determine the numerical values of the parameters of Eq. (7) from the experimental data, the following approximation procedure is adapted to simplify the mathematical computations:

From our estimations, $\lambda_{2}$ is larger than $\lambda_{1}$ by several orders of magnitude, thus, when $t$ is very small,

$$
V(t)=V_{o}+a+\frac{b}{1+\lambda_{2} t}
$$

and for large $t$,

$$
V(t)=V_{o}+\frac{a}{1+\lambda_{1} t}
$$

The table below gives a set of numerical values determined by this procedure and the fitted curves are shown in Figure 8 for the n-channel and in Figure 9 for the p-channel.

Table 2. Values of Annealing Parameters

\begin{tabular}{lllll} 
Sample no. & \multicolumn{1}{l}{3} & 6 & 14 & \multicolumn{1}{c}{16} \\
Specification & \multicolumn{1}{l}{$\mathrm{n}_{\mathrm{B}}$} & $\mathrm{n}_{\mathrm{U}}$ & \multicolumn{1}{c}{$\mathrm{p}_{\mathrm{B}}$} & \multicolumn{1}{c}{$\mathrm{p}_{\mathrm{U}}$} \\
$\mathrm{V}_{\mathrm{o}}$ (volts) & 1.465 & 1.357 & 1.800 & 1.705 \\
$\mathrm{a}\left(10^{-2}\right.$ volts $)$ & 3.003 & 2.379 & 9.446 & 0.655 \\
$\mathrm{M}_{\infty}\left(10^{-2}\right.$ volts $)$ & -1.368 & $\sim 0$ & +2.400 & +6.751 \\
$\lambda_{1}\left(10^{-5} \mathrm{sec}^{-1}\right)$ & 6.066 & 28.420 & 2.166 & 11.230 \\
$\lambda_{2}\left(10^{-3} \mathrm{sec}^{-1}\right)$ & 12.555 & - & 8.333 & 2.083
\end{tabular}

Several interesting features of these numerical values are:

(i) For all devices with different annealing conditions, the values fall in the same orders of magnitude.

(ii) For long-time behavior, the unbiased case is about 5 times faster than the biased case, for all $\mathrm{n}$ and $\mathrm{p}$-channels. 


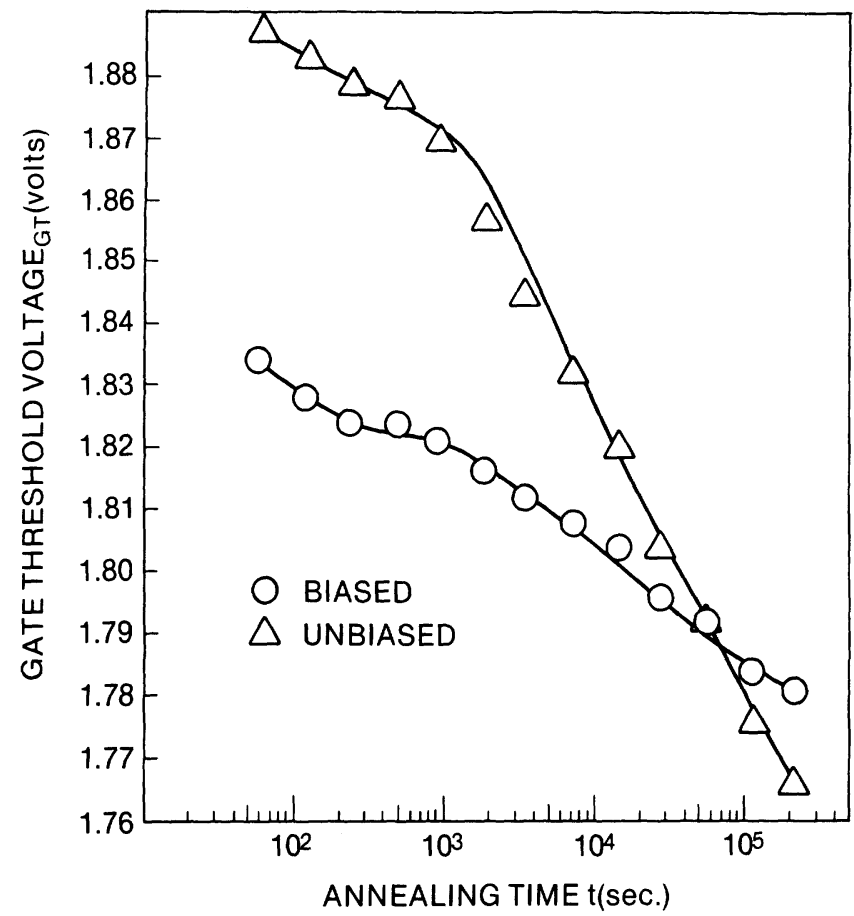

Figure 9. Same as Figure 8, but for p-channels.

(iii) We emphasize once again that $M$ is negative in the case of $n_{B}$, but this is a normal annealing for $n_{B}$. It is a term which gives a reverse annealing. In p-channel case, both a and $b\left(=M_{\infty}\right)$ gives a normal annealing.

In order to compare with a p-channel device made by AMI, for convenience we list in the following a table for this device, ${ }^{1}$

$\begin{array}{ccc} & \mathrm{P}_{\mathrm{B}} & \mathrm{P}_{\mathrm{U}} \\ \lambda_{1}\left(10^{-5} \mathrm{sec}^{-1}\right) & 1.84 & 0.885 \\ \lambda_{2}\left(10^{-3} \mathrm{sec}^{-1}\right) & 48.3 & 83.2\end{array}$

We observe that in the short term, the recovery rate of the AMI device is faster; on the other hand, for the long term recovery, the $Z$-process CMOS is faster. We are especially interested in the shortterm behavior both because of a practical importance and because of its association with a radiation enhanced annealing we proposed earlier. ${ }^{4}$ In this respect, the Z-process CMOS is less advantageous than that of the AMI p-channel.

\section{Acknowledgments}

The authors wish to thank Mr. Lawrence Bromery of the Goddard Space Flight Center Radiation Facility for his valuable assistance and substantial contribution in operating both the $2 \mathrm{MeV}$ Van de Graaff accelerator and Co-60 gamma ray source for these experiments.

\section{References}

1. V. Danchenko, E. G. Stassinopoulos, P. H. Fang and S. S. Brashears, IEEE Trans Nucl. Sci. NS-27, 1658 (1980).

2. R. A. Cliff, V. Danchenko, E. G. Stassinopoulos, M. Sing, G. J. Brucker and R. S. Ohanian, Ibid, NS-23, 1781 (1976).

3. E. G. Stassinopoulos, V. Danchenko, R. A. Cliff, M. Sing. G. J. Brucker and R. S. Ohanian, Ibid, NS-24, 2289 (1977).

4. V. Danchenko, P. H. Fang and S. S. Brashears, "An Anomalous Annealing Characteristic of an MOS Device," to be published. 